\title{
Prevalence of cryptococcal meningitis at a tertiary care centre in Western India (1996-2005)
}

The incidence of cryptococcal meningitis has increased in recent years, both in HIV-positive and -negative patients (Gottfredsonn, 2000). Among all fungi causing meningitis, Cryptococcus neoformans remains the most common, and the cryptococcal antigen is widely recognized as a valuable diagnostic tool in such cases (Lu et al., 2005; Cunha, 2001). The clinical signs and symptoms of $C$. neoformans meningitis are indistinguishable from those of many other causes of meningitis. Early diagnosis of cryptococcal infection is therefore necessary for appropriate management. Although cryptococcosis is generally thought to be associated with immunocompromised patients, such as those with acquired immunodeficiency syndrome (AIDS) and various malignancies (Steenbergen \& Casadevall, 2000), the incidence among immunocompetent patients has reportedly risen over recent years (Hoang et al., 2004; Shih et al., 2000). This retrospective study reports the incidence of cryptococcal meningitis in both HIV and non-HIV patients at a major tertiary healthcare centre in the metropolitan city of Pune, Western India, over the course of 10 years.

During a 10-year period (1996-2005), a total of 2037 samples collected from 1922 clinically diagnosed cryptococcal meningitis cases were processed in the clinical microbiology laboratory of the King Edward Memorial (KEM) Hospital of Pune, a university-affiliated 600-bed teaching general hospital. The laboratory diagnostic criteria were a positive cerebrospinal fluid (CSF) india ink staining or a positive culture for the organisms, in addition to a positive cryptococcal antigen latex agglutination test (CALAS; Meridian Diagnostics). Centrifuged deposits of CSF were cultured on Sabouraud dextrose agar, and blood was cultured in biphasic brain heart infusion medium (Difco).

Cryptococcus was identified on the basis of colony morphology, india ink stain, urease production, nitrate assimilation, sugar assimilation and fermentation tests, as described by Warren et al. (1999). Cryptococcal antigen was tested for in the CSF of all clinically diagnosed patients $(n=1922)$ and in the blood of 115 patients who predominantly had septicaemia rather than meningitis, using CALAS as per the manufacturer's instructions. A titre of $>8$ was considered to be positive for cryptococcal infection; however, a final antigen titre was not determined in all cases. In cases of doubtful and discordant results, CALAS was treated with pronase $B$ to exclude false positivity. Pronase B treatment improves the titre during cryptococcal antigen detection by inhibiting false positivity caused by non-specific factors in the sample, such as rheumatoid factor, heterophilic antibodies, certain infections or false negativity associated with the prozone effect. Normally it does not affect antigen titres in CSF, but increases titres in serum. Biotyping of all isolates was carried out as described by Kwon-Chung et al. (1982). Clinical details concerning the known HIV-positive status of the patients were received from the clinician along with the clinical samples.

A total of 1922 cases were confirmed by laboratory tests for cryptococcal meningitis, through 2037 submitted clinical samples (1922 CSF and 115 blood). About $17 \%$ (340/2037) of the total were found to be positive for Cryptococcus by one or more of the tests. CSF from $15 \cdot 1 \%(290 / 1922)$ of cases was found to be positive when confirmed by CALAS during screening. The results of the CALAS analysis are shown in Table 1. Out of a total of 115 blood samples, $50(43 \cdot 5 \%)$ were confirmed as positive for CALAS. In clinically diagnosed cryptococcosis, $23 \cdot 3 \%(475 / 1922)$ of cases were known to be positive for HIV infection, of which $53 \cdot 1 \%(252 / 475)$ were found positive for cryptococcal meningitis, as confirmed by CALAS positivity. The net incidence of cryptococcosis in HIV-positive patient samples over time is also shown in Table 1. We did not review clinical case records.

Although cryptococcosis is an established disease, its worldwide prevalence was much lower before the AIDS era; however, over the last three decades, its incidence has been continuously rising for various reasons (Hoang et al., 2004). Cryptococcal meningitis has been relatively understudied in developing countries such as India. While cryptococcal meningitis has been reported from the Indian subcontinent, showing a high prevalence (Chakrabarti et al., 2000; Madan et al., 1999), we did not observe any significant rise in our study over the time period chosen. The overall prevalence remained between 9 and $27 \%$ (Table 1), and no specific trend of increase in incidence was discernible during this time. These findings are comparable with those of the earlier report of Shih et al. (2000).

Although C. neoformans var. gatti has been reported from India (Padhye et al., 1993), we did not find it in our patient population, and all isolates were $C$. neoformans var. neoformans. During the study period, the prevalence of cryptococcal meningitis in HIV-positive patients was between 49 and $100 \%$ (Table 1). Although no significant increase was observed such as that for nonHIV patients, the incidence of cryptococcal meningitis reached $100 \%$ in the years 1998 (39/39 cases) and 2003 (20/20 cases) and $87 \%$ in 2005 (26/30 cases), which is very high. To our knowledge, there is no report in which $100 \%$ of HIV-positive patients were infected with Cryptococcus and developed meningitis. However, when we compare the results in terms of the total number of known HIV-positive cases included, the incidence comes down to about $62 \%$ (1998), $59 \%$ (2003) and $63 \%$ (2005), although this is still higher than most recent reports (Bekondi et al., 2006). Cryptococcal meningitis is generally considered rare in immunocompetent patients and therefore specific treatment is not implemented until the organism is identified or cryptococcal antigen detected. 
Table 1. Detection of cryptococcal antigen in CSF and blood from clinically suspected patients by the latex agglutination method

Positive columns show the number (percentage) of cryptococcal antigen-positive samples. Also shown is the incidence of cryptococcal meningitis (CM) (in \%) in HIV and non-HIV patients as a proportion of the total number of CM cases.

\begin{tabular}{|c|c|c|c|c|c|c|c|c|c|c|}
\hline \multirow[t]{2}{*}{ Year } & \multicolumn{2}{|c|}{ All samples (\%) } & \multicolumn{2}{|c|}{ CSF (\%) } & \multicolumn{2}{|c|}{ Blood (\%) } & \multicolumn{2}{|c|}{ Known HIV positive (\%) } & \multicolumn{2}{|c|}{ Percentage CM positive } \\
\hline & Total & Positive & Total & Positive & Total & Positive & Total & Positive & HIV & Non-HIV \\
\hline 1996 & 70 & $15(21 \cdot 4)$ & 58 & $10(17 \cdot 2)$ & 12 & $5(41 \cdot 7)$ & 9 & $8(88 \cdot 9)$ & 53 & 47 \\
\hline 1997 & 140 & $30(21 \cdot 4)$ & 125 & $24(19 \cdot 2)$ & 15 & $6(40)$ & 47 & $17(36 \cdot 2)$ & 57 & 43 \\
\hline 1998 & 219 & $39(17 \cdot 8)$ & 212 & $37(17 \cdot 5)$ & 7 & $2(28 \cdot 6)$ & 63 & $39(61 \cdot 9)$ & 100 & 0 \\
\hline 1999 & 268 & $55(20 \cdot 5)$ & 245 & $42(17 \cdot 1)$ & 23 & $13(56 \cdot 5)$ & 59 & $27(45 \cdot 8)$ & 49 & 51 \\
\hline 2000 & 261 & $71(27 \cdot 2)$ & 246 & $64(26)$ & 15 & $7(46 \cdot 7)$ & 92 & $55(59 \cdot 8)$ & 77 & 23 \\
\hline 2001 & 240 & $36(15)$ & 235 & $33(14)$ & 5 & $3(60)$ & 54 & $30(55 \cdot 6)$ & 83 & 17 \\
\hline 2002 & 163 & $15(9 \cdot 2)$ & 153 & $14(9 \cdot 2)$ & 10 & $1(10)$ & 36 & $11(30 \cdot 6)$ & 73 & 27 \\
\hline 2003 & 214 & $20(9 \cdot 3)$ & 209 & $18(8 \cdot 6)$ & 5 & $2(40)$ & 34 & $20(58 \cdot 8)$ & 100 & 0 \\
\hline 2004 & 231 & $29(12 \cdot 6)$ & 221 & $25(11 \cdot 6)$ & 10 & $4(40)$ & 40 & $19(47 \cdot 5)$ & 66 & 34 \\
\hline 2005 & 231 & $30(13 \cdot 0)$ & 218 & $23(10 \cdot 6)$ & 13 & $7(53 \cdot 8)$ & 41 & $26(63 \cdot 4)$ & 87 & 13 \\
\hline Total & 2037 & $340(16 \cdot 7)$ & 1922 & $290(15 \cdot 1)$ & 115 & $50(43 \cdot 5)$ & 475 & $252(53 \cdot 1)$ & - & - \\
\hline
\end{tabular}

Since the clinical manifestations and the results of CSF analysis (routine and biochemistry) for cryptococcal meningitis are similar to those of tubercular meningitis, viral meningitis and atypical purulent meningitis, the rapid diagnosis of cryptococcal meningitis depends on detection of the cryptococcal antigen in tests such as CALAS (for serum or CSF).

Cryptococcal antigen detection is a highly specific and rapid test, and the antigen can remain detectable for several months after infection (Lu et al., 2005). It

is therefore a suitable choice of laboratory test for screening.

\section{Meena G. Satpute, Nilima V. Telang, Geetanjali M. Litake, Krishna B. Niphadkar and Suresh G. Joshi $†$}

Department of Clinical Microbiology, King Edward Memorial (KEM) Hospital, Sardar Mudliar Road, Pune 411011 , India

tPresent address: Division of Infectious Diseases, Department of Medicine, Thomas Jefferson University Medical College, 1020 Locust Street, Philadelphia, PA 19107, USA.
Correspondence: Suresh G. Joshi

(Suresh.Joshi@Jefferson.edu)

Bekondi, C., Bernede, C., Passone, N., Minssart, P., Kamalo, C., Mbolidi, D. \& Germani, Y. (2006). Primary and opportunitistic pathogens associated with meningitis in adults in Bangui, Central African Republic, in relation to human immunodeficiency virus serostatus. Int J Infect Dis (in press; http://dx.doi.org/10.1016/j.ijid.2005.07.004).

Chakrabarti, A., Sharma, A., Sood, A., Grover, R., Sakhuja, V., Prabhakar, S. \& Verma, S. (2000). Changing scenario of cryptococcosis in a tertiary care hospital in north India. Indian J Med Res 112, 56-60.

Cunha, B. A. (2001). Central nervous system infections in the compromised host: a diagnostic approach. Infect Dis Clin North Am 15, 567-590.

Gottfredsonn, M. (2000). Fungal meningitis. Semin Neurol 20, 307-322.

Hoang, L. M. N., Maguire, J. A., Doyle, P., Fyfe, M. \& Roscoe, D. L. (2004). Cryptococcal neoformans infections at Vancouver Hospital and Health Sciences Center (1997-2002): epidemiology, microbiology and histopathology. J Med Microbiol 53, 935-940.

Kwon-Chung, K. J., Polacheck, I. \& Bennet, J. (1982). Improved diagnostic medium for separation of Cryptococcus neoformans var. neoformans (serotypes A and D) and Cryptococcus neoformans var. gattii (serotypes B and C). J Clin Microbiology 15, 535-537.

Lu, H., Zhou, Y., Yin, Y., Pan, X. \& Weng, X. (2005). Cryptococcal antigen test revisited: significance for cryptococcal meningitis therapy monitoring in a tertiary Chinese hospital. J Clin Microbiol 43, 2989-2990.

Madan, M., Ranjitham, M., Chandrasekharan, S. \& Sudhakar (1999). Cryptococcal meningitis in immunocompetent individuals. J Assoc Physicians India 47, 933-934.

Padhye, A. A., Chakrabarti, J., Chander, J. \& Kaufman, L. (1993). Cryptococcus neoformans var. gattii in India. J Med Vet Mycol 31, 165-168.

Shih, C. C., Chen, Y. C., Chang, S. C., Luh, K.-T. \& Hsieh, W.-C. (2000). Cryptococcal meningitis in non-HIV-infected patients. Q J Med 93, 245-251.

Steenbergen, J. N. \& Casadevall, A. (2000).

Prevalence of Cryptococcus neoformans var. neoformans (serotype D) and Cryptococcus neoformans var. grubii (serotype A) isolates in New York City. J Clin Microbiol 38, 1974-1176.

Warren, N. G. \& Hazen, K. C. (1999). Candida, Cryptococcus and other yeasts of medical importance. In Manual of Clinical Microbiology, pp. 1184-1199. Edited by P. R. Murray, E. J. Baren, M. A. Pfaller, F. C. Tenover \& R. H. Yolken. Washington DC: American Society for Microbiology. 